\title{
Research on 3-D laser-scanning-based machine vision system for robotic construction vehicles
}

\author{
Yan SONG ${ }^{1, \mathrm{a}}$, Dewen $\mathrm{KONG}^{2, \mathrm{~b}, *}$, and ${\mathrm{Zhi} \mathrm{LI}^{2}}^{2}$ \\ ${ }^{1}$ College of Biological and Agricultural Engineering \\ Jilin University, Changchun, China \\ ${ }^{2}$ College of Mechanical Science and Engineering \\ Jilin University, Changchun, China \\ a 451192221@qq.com, ${ }^{\text {b }}$ dwkong@jlu.edu.cn \\ *corresponding author
}

Keywords: Laser scanning, Laser rangefinder, Machine vision, Robotic construction vehicle

\begin{abstract}
Based on the AR4000-LIR type infrared laser rangefinder, the prototype of the 3-D laserscanning-based machine vision system for robotic construction vehicles is developed and the software for scanning control, swing control and data sampling/processing is programmed. The experimental results show that the system has good spatial resolution and stability and is able to be used for the task planning of the robotic construction vehicles which is in autonomous operation.
\end{abstract}

\section{INTRODUCTION}

Wheel loaders and excavators are commonly used construction vehicles. The autonomous operation technology of the construction vehicle is increasingly drawing the attention of the worldwide researchers, and some results have been achieved, such as the autonomous robotic excavator developed at Carnegie - Mellon University, the automatic working efficiency of the experimental prototype being close to the level of skilled operators [1].

About $80 \%$ of the perceptual information of the human world is acquired through vision, so the vision system is an integral part of intelligent machines. Commonly used machine vision methods are stereo vision, structured light and laser scanning. The robotic construction vehicle works in unstructured complex environment, and the 3D laser scanning method is able to obtain accurate surrounding environmental information in real time, so this method has a good application prospect.

As the vision system of an intelligent vehicle, the 3D laser scanning system has two kinds of application modes: one is that the 3D laser scanning system directly installed on the construction vehicle to get the job information in real time, such as the pile material varying with the operation for truck loading; the other is that the 3D laser scanning system is installed in the proper position of the ground to obtain the space position information of the construction vehicle, stockpile and transport vehicles. These two methods can realize the real-time task planning for the autonomous operation of the robotic construction vehicle. The advantage of the former approach is that the vehicle vision system and the laser scanning system are in one coordinate system, so as to the amount of data processing being smaller, but the laser scanning vision system is subject to being affected by the vibration of the vehicle in operation; the latter approach is that the laser scanning system is placed in the global coordinate system, so that the operation of the construction vehicle and the operation target are need to be scanned at the same time, leading to a large amount of data processing, but being not affected by the vibration of the construction vehicle.

\section{The Configuration and Working principle of the 3D Laser Vision System}

The core of the system is an AR4000-LIR type infrared laser ranging sensor, namely laser rangefinder, and its front end is equipped with a scanning mirror which can rotate in 360 degrees. 
The scanning mirror is mounted at 45 degrees with the laser axis, and is driven by a DC motor with a photoelectric encoder to achieve line scanning of the target being measured. The laser and scanning mirror are integrally packaged in the case to realize dust proof and vibration isolation. The whole system is installed on the NC rotary worktable, driven by a step motor controlled by an industrial computer to rotate in 360 degrees, so as to realize the 3D scanning of the surrounding scenes. A digital $\mathrm{I} / \mathrm{O}$ card installed in an industrial computer is programmed to generate a rectangular pulse signal and is driven by a step motor controller to rotate the NC turntable. The high-speed data acquisition card is also used to collect ranging information from the laser sensor and the angular information of the encoder while driving the scanning motor to rotate at high speed. The acquisition card also acquires the temperature information of the sensor and the brightness information of the target object. The system configuration is shown in Figure 1.

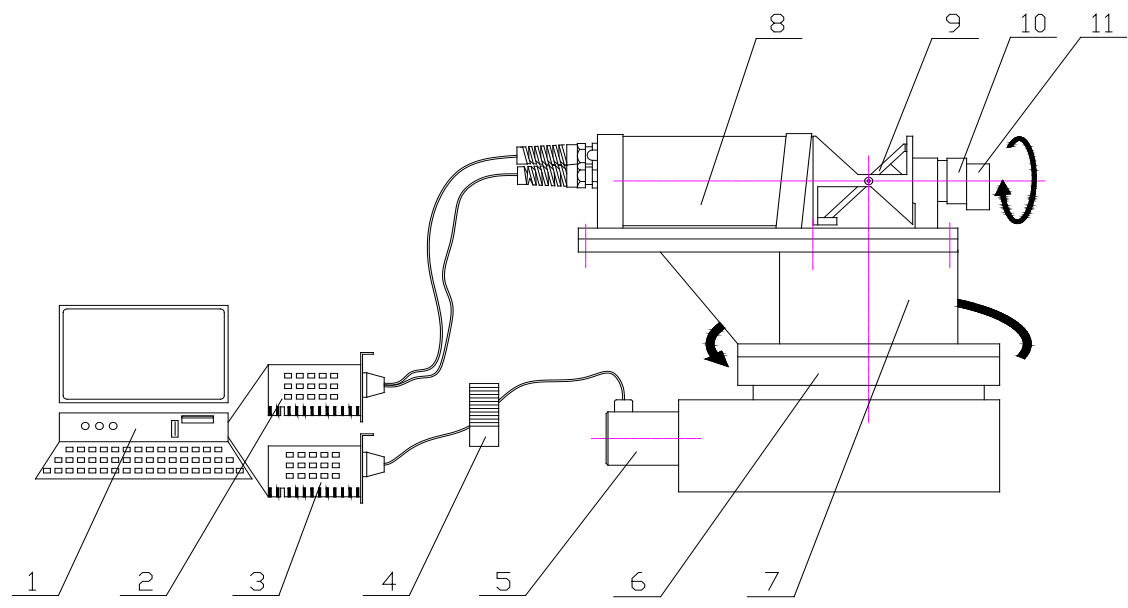

Figure 1 Configuration of the 3-D Laser Scanning System 1 - Industrial computer, 2 - Scanning control and data acquisition card, 3 - Digital I/O card, 4 - Step motor controller, 5 - Step motor, 6 - CNC turntable, 7 - Bracket, 8 - Laser range finder, 9 - Scanning mirror, 10 - DC motor, 11 - Encoder

The main technical parameters of the system are as follows:

Laser sensor wavelength: 780nm

Laser sensor power: $20 \mathrm{~mW}$

Effective measuring distance: $15.24 \mathrm{~m}$ (50 ft.)

Maximum measurement distance accuracy: $2.54 \mathrm{~mm}$ (0.1 inches)

Scanning mirror reflectivity: $96 \%$

Scanning drive motor maximum speed: $2600 \mathrm{r} / \mathrm{min}$

Photoelectric encoder performance: 2000 count / turn

High speed interface card sampling rate: $50 \mathrm{kHz}$

Indexing accuracy of CNC turntable: 30 "

\section{Scanning Control and Program Implementation}

The scanning control software is programmed by Visual $\mathrm{C}++6$, which is mainly used to realize the drive control of the scanning mirror, the turntable rotation control and data acquisition.

\subsection{Scan Mirror Drive Control}

The driving motor of scanning mirror is controlled by AR4000 high-speed interface and PCI HSIF card. The drive motor needs to be connected with the DC power supply of 12 to $15 \mathrm{~V}$, and the driving voltage is divided into 256 voltage levels by the high-speed interface card according to the control instruction, so as to realize the speed control of the drive motor. The following motor control subroutine accelerates the motor speed from 0 to full speed and then slows down to 0 . The test subroutine is as follows: 


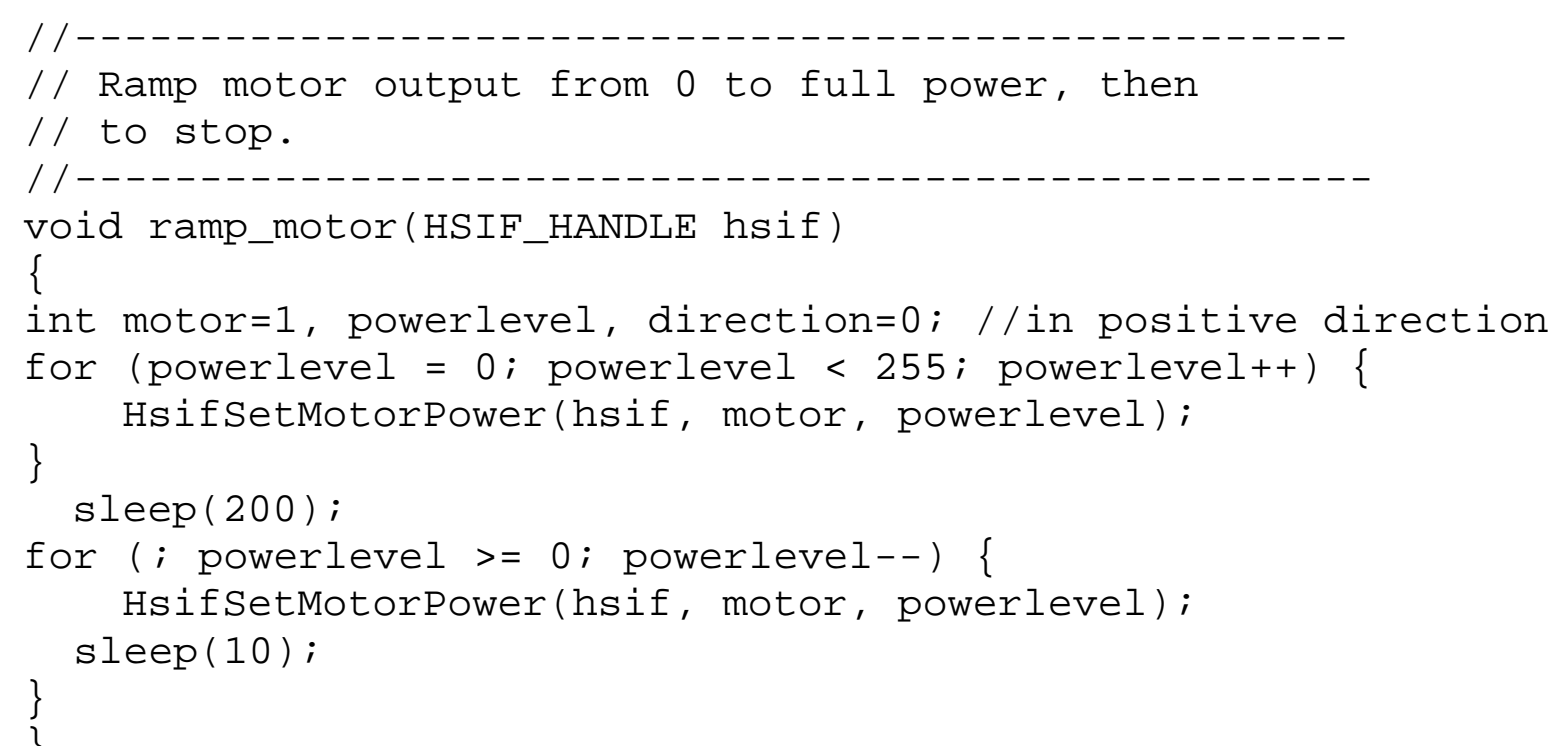

The HsifSetMotorPower() function in the program is from the motor drive control function library provided by the manufacturer.

\subsection{The Turntable Swing Control}

The rotary control of the turntable is transmitted to the step motor controller through the photoelectric isolation digital I/O card PCI7606, and then the turntable is driven by the step motor controller. The stepped-angle of the step motor controller is adjustable from 0.036 to 0.9 degrees. The gear ratio of the NC turntable is 180 , so the theoretical step angle of the turntable is between 0.0002 and 0.005 degrees, i.e., $0.72 "$ to 18 ". However, the indexing accuracy of the turntable is only 30 ", which is lower than the theoretical stepped-angle. Because the laser ranging sensor has a signal cable, the turntable rotates within less than 360 degrees for reciprocating swing in actual use, and the scan line indexing angle is determined according to the scanning precision. The 0.1 degree is taken for the test in this paper. Turntable rotation control program is as follows:

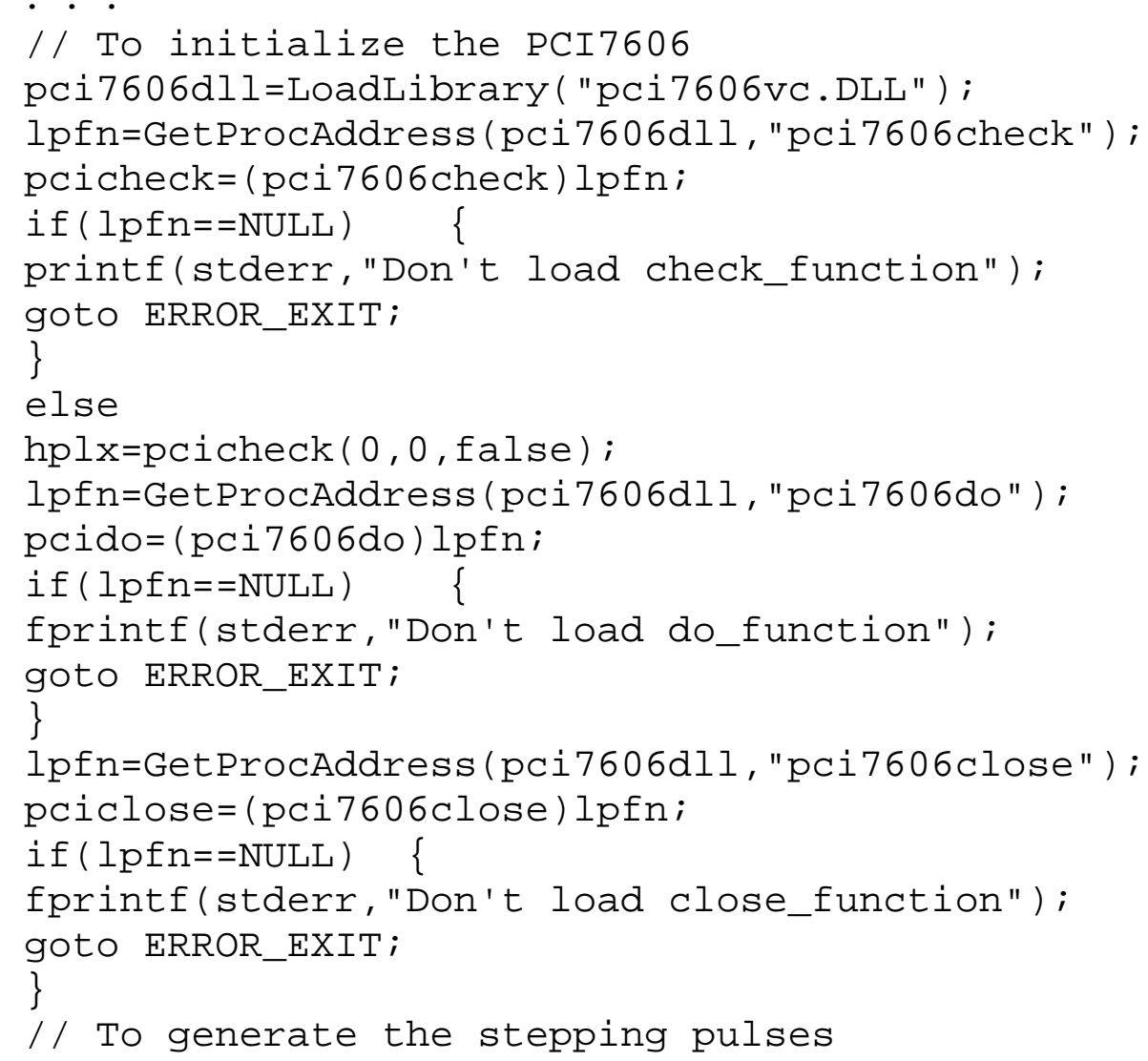




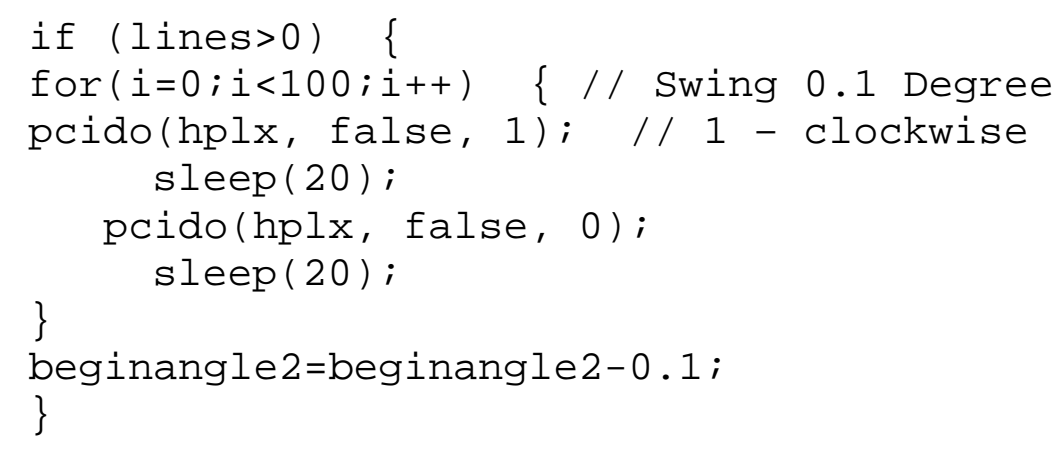

\subsection{Scan Data Acquisition}

The data acquisition of this system is realized by AR4000 high speed interface PCI HSIF card. The interface card can get the sample data with 16 bytes format through a data bus, including one segment of 32 bit distance information and two segments of 32 bit scanning mirror encoder information and other information of a byte (object brightness, ambient light intensity and temperature sensor etc.). Data acquisition procedures are as follows:

// Header ignored...

main(int argc, char *argv[]) \{

// Variable definition ignored...

//To open sampling data file

if $((\mathrm{fp}=$ fopen ( "cap. txt", "w") )==NULL $)\{$

fprintf(stderr,"Cannot open file. In");

\} goto ERROR_EXIT;

// To initialize PCI HSIF card and PCI 7606 card, ignored. .

// To start the scan mirror drive motor

HsifSetMotorPower(hsif, 2, 100);

sleep (5000);

//To get in the sampling loop

while(lines--) \{ // sample hsif, calculate and print distances, // To initialize the sampling buffer and data communication

//port, ignored...

// To read the sample data

trap_count $=0$;

for (trap_count $=0$, sample $=0$;

sample<nsamples; ) \{

// blocking call if last parameter set to TRUE

result=HsifGetBufferedSamples(hsif,

hsbuffer+sample, nsamples-sample, \&numRead, TRUE);

if (result == HSIF_OVERFLOW)

fprintf(stderr,"Overflow $\backslash n$ ");

sample $+=$ numRead;

if(trap_count++>=100) \{

fprintf(stderr,"Timeout waiting for samples $\backslash n ")$;

\} break;

\}

Sleep (1);

numRead = sample;

//To read the scan mirror encoder data

for $(i=0 ; i<$ numRead $; i++)$

hsbuffer [i] . encoder1=(hsbuffer [i] . encoder 1$) \% 4096$;

// To process the sampled results 


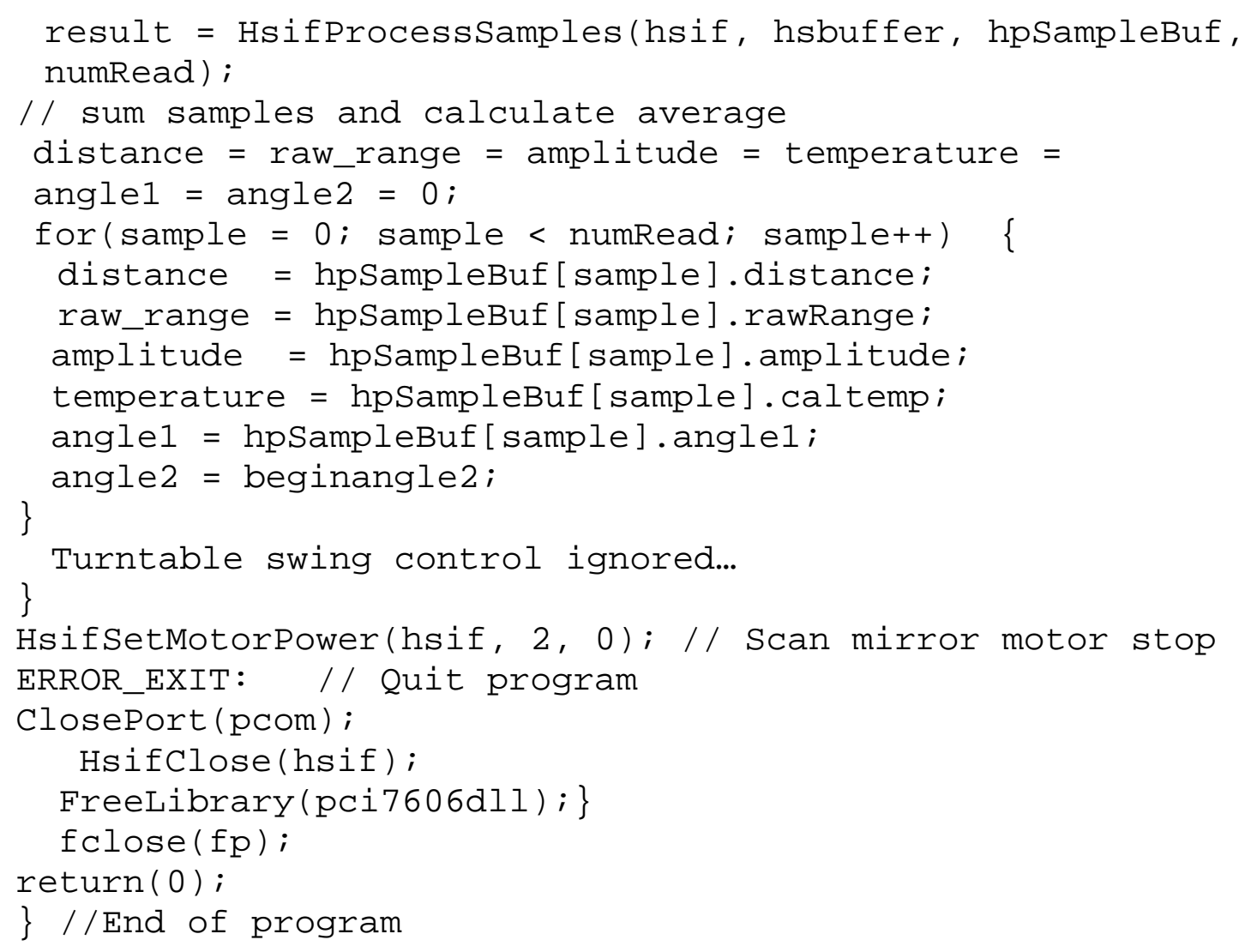

\section{Scanning Test and Data Processing}

The object used in the test is a plastic bottle with a stereoscopic relief pattern on its surface and is scanned at a distance of about 1 meter. Figure 2 shows a 3D contour map recovered from raw data that has never been denoised. The 3D profile obtained by smoothing filtering is shown in figure 3 .

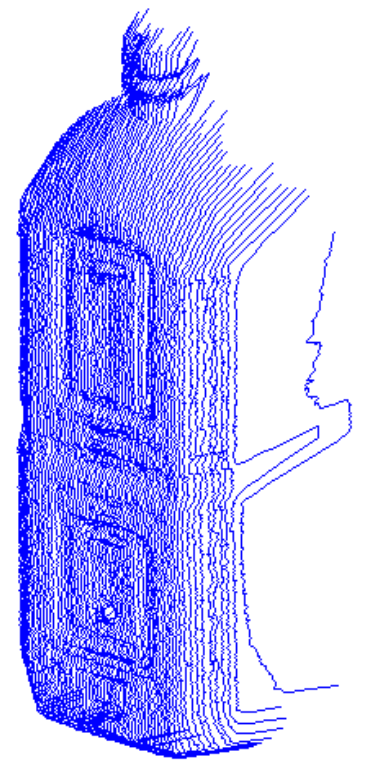

Figure 2. Contour from Raw Scanning Data

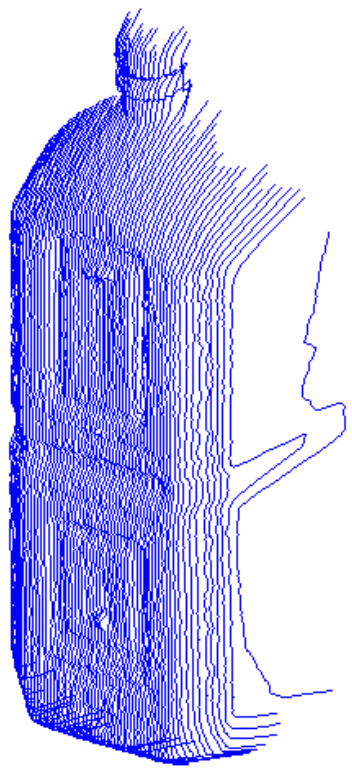

Figure 3. Contour from Denoised Scanning Data

\section{Conclusion and Further Research Prospect}

This paper describes the research and development of a 3D laser ranging system based on AR4000-LIR laser vision sensor, intended for robotic vehicle autonomous operation, the scanning 
control and data acquisition and processing program. The experimental results show that the system has high scanning accuracy and stability, and can recover the detail contours of scanned objects. After further research and further improvement, the system can be used as a visual system for automatic operation of the robotic construction vehicles. Further research efforts should include:

(1) Miniaturization of the scanning controller;

(2) Research on anti-dithering technology of laser scanning system;

(3) Real-time data processing algorithm.

\section{References}

[1] Anthony Stentz, John Bares, Sanjiv Singh and Patrick Rowe, A Robotic Excavator for Autonomous Truck Loading, Autonomous Robots 7, 175-186 (1999).

[2] Acuity Research Incorporated, AccuRange High Speed Interface \& AccuRange Line Scanner User’s Manual, Jan. 25, 2000.

[3] Zhi Li, Research on 3D machine vision system based on laser scanning [D], May, 2007.

[4] AccuRange High Speed Interface PCI Formats User’s Manual, July, 2005.

[5] CTI-HSIF Software Library for Acuity Research Inc.AR4000 Laser Distance Sensors Programmer’s Guide and Reference Manual.2002. 